\title{
THE ANTIBIOTIC W341C, ITS ION TRANSPORT PROPERTIES AND INHIBITORY EFFECTS ON MITOCHONDRIAL SUBSTRATE OXIDATION ${ }^{\dagger}$
}

\author{
Robert S. Wehbie, Cai Runsheng ${ }^{\dagger \dagger}$ and Henry A. Lardy* \\ Institute for Enzyme Research and Department of Biochemistry, \\ University of Wisconsin, \\ Madison, Wisconsin 53705, U.S.A.
}

(Received for publication February 9, 1987)

\begin{abstract}
We have examined the ion transport properties and the inhibition of rat liver mitochondrial substrate oxidation by the antibiotic W341C. W341C was able to transport ${ }^{22} \mathrm{Na}^{+}$ and ${ }^{42} \mathrm{~K}^{+}$across a bulk carbon tetrachloride layer. A preference was shown for $\mathrm{K}^{+}$transport. With equal molar antibiotic concentrations, W341C transported ${ }^{42} \mathrm{~K}^{+}$at a greater rate than the $\mathrm{K}^{+}$-selective ionophore nigericin, but transported ${ }^{22} \mathrm{Na}^{+}$at a lesser rate than the $\mathrm{Na}^{+}$selective ionophore monensin. Like nigericin, W341C was able to deplete mitochondrial $\mathrm{K}^{+}$, but not $\mathrm{Mg}^{2+}$ nor $\mathrm{Ca}^{2+}$. The inhibition of mitochondrial substrate oxidation by W341C paralleled the patterns obtained with nigericin. These data indicate that $\mathrm{W} 341 \mathrm{C}$ is a $\mathrm{K}^{+}$. selective ionophore that inhibits mitochondrial substrate oxidation by a mechanism analogous to that of nigericin.
\end{abstract}

Antibiotic W341C is a monocarboxylic polyether antibiotic with anticoccidal properties ${ }^{22}$ produced by Streptomyces W341.3) Previous work demonstrated the ability of W341C to induce potassium loss in Bacillus subtilis and Streptococcus lactiae, and promote potassium uptake into Escherichia coli. ${ }^{4)}$ The structure of W341C is shown in Fig. 1.5)

In the present study, the ion transport properties of $\mathrm{W} 341 \mathrm{C}$ are examined, with particular reference to inhibitory effects of this antibiotic on mitochondrial ion release and substrate oxidation.

\section{Materials and Methods}

\section{Materials}

Materials were obtained from the following sources: ${ }^{22} \mathrm{NaCl},{ }^{42} \mathrm{KCl}$, and Aquasol, New England Nuclear; $\left[8-{ }^{14} \mathrm{C}\right] \mathrm{ADP}$ and $\left[U-{ }^{14} \mathrm{C}\right]$ sucrose, Amersham; carboxyatractyloside, Boehringer Mannheim; carbonylcyanide $p$-trifluoromethoxyphenylhydrazone (FCCP), Calbiochem; A23187 and monensin were gifts of Dr. R. HAMILL, Eli Lilly and nigericin was a gift of Dr. R. HARNED, Commercial Solvents Company. DC 200 and DC 500 are Dow Corning silicone oils obtained from William F. Nye, Inc.

Fig. 1. Structure of antibiotic W341C.4)

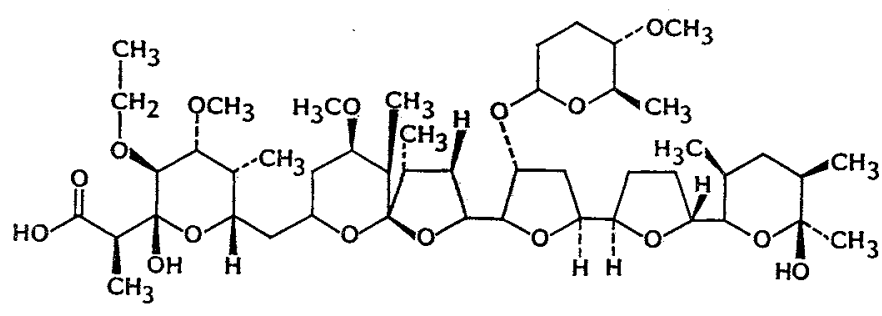

$t$ Portions of this work were presented in preliminary form. ${ }^{12}$

it Shanghai Institute Materia Medica, Shanghai, China. 
All other materials were of the highest purity available from commercial sources.

Antibiotic W341C

W341C was isolated at the Shanghai Institute Materia Medica, Shanghai, China as described previously. ${ }^{3)}$

\section{Measurement of Bulk Phase Cation Transport}

Antibiotic-mediated cation transport across a bulk phase was assessed as described by Pressman. ${ }^{\text {b) }}$ The system employed consisted of a glass chamber with a middle partition separating two top aqueous compartments of $1.0 \mathrm{ml}$ each from a common, bottom $1.7 \mathrm{ml} \mathrm{CCl}$ layer (interphase of $73 \mathrm{~mm}^{2}$ ). The antibiotic to be tested was dissolved in the $\mathrm{CCl}_{4}$ layer; which was stirred with a magnetic bar. After allowing the chamber to equilibrate for 1 hour, ${ }^{22} \mathrm{NaCl},{ }^{42} \mathrm{KCl}$ or $\mathrm{MgCl}_{2}$ was added to one aqueous compartment, and aliquots were removed from the opposite aqueous compartment at hourly intervals. No ion transport was observed when the organic layer was antibiotic-free.

\section{Mitochondria}

Rat liver mitochondria were isolated according to the procedure of JOHNSON and LARDY ${ }^{7)}$ in $0.25 \mathrm{~m}$ mannitol, $70 \mathrm{~mm}$ sucrose and $10 \mathrm{~mm}$ 4-(2-hydroxyethyl)-1-piperazineethanesulfonic acid (HEPES) adjusted to $\mathrm{pH} 7.4$ with triethanolamine (TEA). The homogenization medium contained $1.0 \mathrm{~mm}$ ethyleneglycol-bis-( $\beta$-aminoethyl ether)- $N, N^{\prime}$-tetraacetic acid (EGTA). Protein concentration was determined by the biuret reaction on deoxycholate-solubilized mitochondrial samples. ${ }^{8)}$ Bovine serum albumin was used as the protein standard. Mitochondrial respiration was monitored at $30^{\circ} \mathrm{C}$ using a Clark oxygen electrode.

Preparation of Mitochondria for Potassium and Arsenate Analysis

$400 \mu \mathrm{l}$ aliquots of mitochondrial suspension were added to Eppendorf tubes containing $300 \mu 1$ of a silicone oil mixture of $8.5 \%$ DC 200 and $91.5 \%$ DC 500 . The tubes were then centrifuged for 30 seconds at $15,600 \times g$. An aliquot of the aqueous layer was removed for $\mathrm{K}^{+}$determination and the remainder of the aqueous layer, as well as a portion of the silicone oil layer, was aspirated from the tube. The sides of the tubes were then wiped with a cotton swab. Next, $300 \mu \mathrm{l}$ of $12 \%$ TCA were added to the tubes, which were then vigorously vortexed to disperse the mitochondrial pellets. At this point, the tubes were recentrifuged to yield a top silicone oil layer and a bottom aqueous layer. Arsenate concentrations were determined on aliquots removed from the aqueous layer. Mitochondrial arsenate contents were corrected for non-matrix space arsenate by subtracting the mitochondrial arsenate content obtained from parallel experiments which included $10 \mathrm{~mm}$ mersalyl, an inhibitor of phosphate uptake in mitochondria. ${ }^{9)}$ No stimulation of respiration by arsenate was observed with this concentration of mersalyl using a variety of substrates (data not shown).

\section{Measurement of Ion Concentrations}

${ }^{22} \mathrm{Na}^{+}$and ${ }^{42} \mathrm{~K}^{+}$concentrations were determined with a Nuclear Chicago gamma counter. $\mathrm{Mg}^{2+}$, $\mathrm{K}^{+}$and $\mathrm{Ca}^{2+}$ concentrations were determined by atomic absorption using a Perkin-Elmer spectrophotometer 403.

Arsenate concentrations were determined with the malachite green-inorganic phosphate assay method of LANZETTA, et al. ${ }^{10)}$ Standard curves obtained with this assay using arsenate were identical to those obtained with phosphate as a standard.

\section{Results}

As an initial evaluation of W341C's ion transport properties, the ability of this antibiotic to convey cations across a $\mathrm{CCl}_{4}$ layer was compared to that of several well-characterized ionophores. As shown in Fig. 2, W341C was able to transport both $\mathrm{K}^{+}$and $\mathrm{Na}^{+}$through the organic phase; with the rate of transport being greater for $\mathrm{K}^{+}$. With equimolar antibiotic concentrations, W341C transported $\mathrm{K}^{+}$ at a slightly greater rate than the $\mathrm{K}^{+}$-selective ionophore nigericin, but transported $\mathrm{Na}^{+}$at a lesser rate 
Fig. 2. Ionophore-mediated bulk phase $\mathrm{Na}^{+}$and $\mathrm{K}^{+}$transport.

Bulk phase ion transport was measured as described under Materials and Methods. $50 \mathrm{nmol}$ of the antibiotic to be tested were dissolved in the $\mathrm{CCl}_{4}$ layer. The aqueous layers were buffered with 5.0 mM HEPES-TEA, pH 7.4. ${ }^{22} \mathrm{Na}^{+}(75 \mu \mathrm{Ci} /$ $\mathrm{mmol})$ and ${ }^{42} \mathrm{~K}^{+}(400 \mu \mathrm{Ci} / \mathrm{mmol})$ were added to a final concentration of $10 \mathrm{~mm}$.

$\bigcirc \mathrm{W} 341 \mathrm{C}+\mathrm{K}^{+}, \mathrm{W} 341 \mathrm{C}+\mathrm{Na}^{+}, \square$ nigericin + $\mathrm{K}^{+}, \triangle$ monensin $+\mathrm{Na}^{+}$.

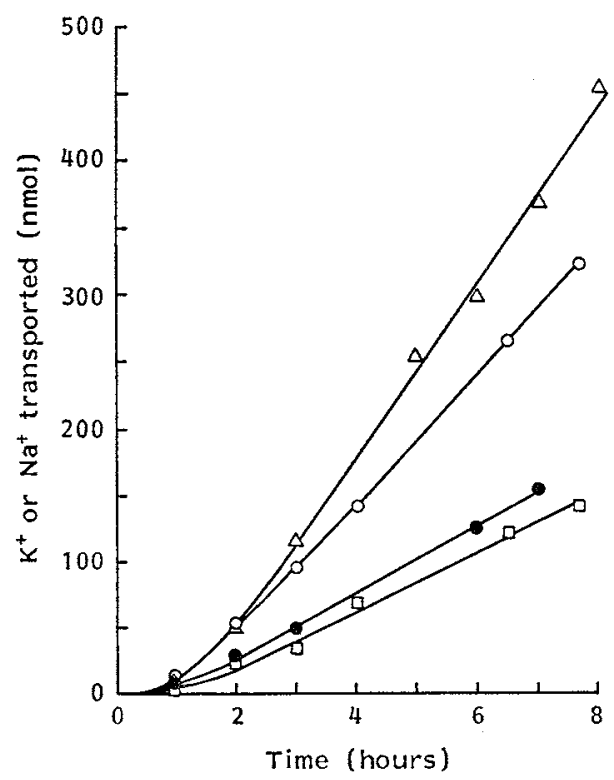

Fig. 3. Ionophore-mediated bulk phase $\mathrm{Mg}^{2+}$ transport.

Bulk phase ion transport was measured as described under Materials and Methods. $800 \mathrm{nmol}$ of the antibiotic to be tested were dissolved in the $\mathrm{CCl}_{4}$ layer. One aqueous layer contained $10.0 \mathrm{mM}$ $\mathrm{MgCl}_{2}$ and $5.0 \mathrm{~mm}$ HEPES-TEA, pH 8.0. The other aqueous layer was buffered at $\mathrm{pH} 5.0$ with 10.0 mM EDTA.

W341C, $\square$ A23187.

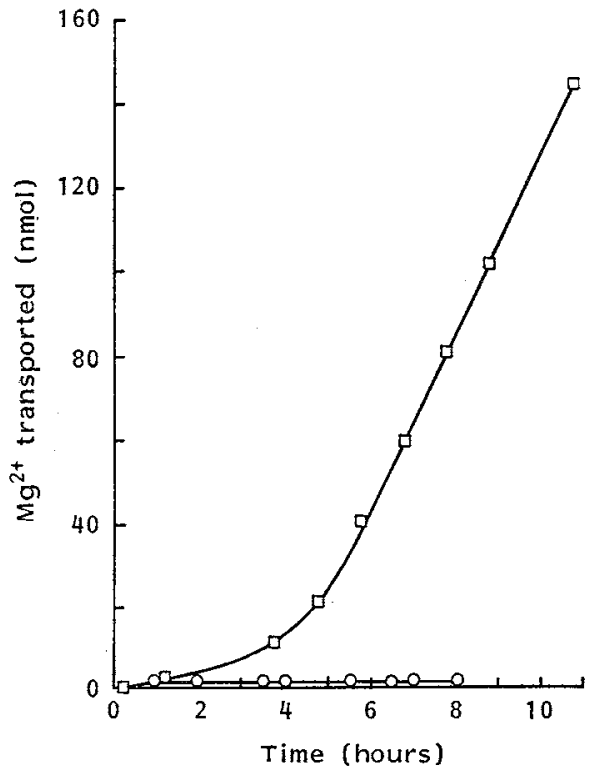

Table 1. Antibiotic induced ion release from mitochondria.

\begin{tabular}{lccrr}
\hline \multirow{2}{*}{ Ion } & \multicolumn{4}{c}{ Amount released to supernatant (nmol/mg protein) } \\
\cline { 2 - 5 } & Control & A23187 & W341C & Nigericin \\
\hline $\mathrm{K}^{+}$ & $39 \pm 1$ & $37 \pm 2$ & $179 \pm 5$ & $176 \pm 1$ \\
$\mathrm{Mg}^{2+}$ & $9 \pm 1$ & $14 \pm 1$ & $10 \pm 1$ & $9 \pm 1$ \\
$\mathrm{Ca}^{2+}$ & $3 \pm 1$ & $8 \pm 1$ & $4 \pm 1$ & $3 \pm 1$ \\
\hline
\end{tabular}

Mitochondria $\left(8.9 \mathrm{mg}\right.$ protein) were incubated in a total volume of $1.2 \mathrm{ml}$ at $30^{\circ} \mathrm{C}$ for 30 seconds in $0.25 \mathrm{~m}$ mannitol, $70 \mathrm{~mm}$ sucrose, $0.5 \mathrm{~mm}$ EDTA, $10 \mathrm{mM}$ HEPES-TEA, pH 7.4, and $1 \mu \mathrm{M}$ of the antibiotic indicated. Incubations were terminated by centrifugation for 2 minutes at $8,000 \times g$. The amount of ion released to the supernatant was determined. Total mitochondrial $\mathrm{K}^{+}, \mathrm{Mg}^{2+}$ and $\mathrm{Ca}^{2+}$ were $420 \pm 45$, $43 \pm 1$ and $10 \pm 2 \mathrm{nmol} / \mathrm{mg}$ protein, respectively. Results are expressed as the mean $\pm \mathrm{SD}$ of triplicates from one representative experiment.

than the $\mathrm{Na}^{+}$-selective ionophore monensin. However, W341C was unable to transport $\mathrm{Mg}^{2+}$ in contrast to the $\mathrm{Mg}^{2+} / \mathrm{Ca}^{2+}$ ionophore A23187 (Fig. 3).

To test the ability of $\mathrm{W} 341 \mathrm{C}$ to deplete mitochondrial potassium, magnesium and calcium, mitochondria were incubated with equimolar concentrations of W341C, nigericin or A23187. As shown in Table $1, \mathrm{~W} 341 \mathrm{C}$ behaved similarly to nigericin in its ability to extract approximately $40 \%$ of the mitochondrial potassium. No significant effect of $\mathrm{W} 341 \mathrm{C}$ on mitochondrial magnesium or calcium 
Table 2. Inhibition of mitochondrial substrate oxidation by W341C.

\begin{tabular}{lcc}
\hline & \multicolumn{2}{c}{ Inhibition (\%) } \\
\cline { 2 - 3 } \multicolumn{1}{c}{ Substrate } & State 3 & $\begin{array}{c}\text { FCCP- } \\
\text { uncoupled }\end{array}$ \\
\hline Caprylate $(20 \mu \mathrm{M})$ & 100 & 100 \\
Pyruvate & 100 & 100 \\
Malate & 100 & 100 \\
Proline & 89 & 88 \\
Glutamate & 100 & 100 \\
Malate+pyruvate & 100 & 100 \\
Malate+proline & 100 & 97 \\
Malate+glutamate & 97 & 0 \\
Succinate & 94 & 13 \\
L-Glycerol-3-phosphate & 78 & 14 \\
B-Hydroxybutyrate & 100 & 18 \\
\hline
\end{tabular}

Mitochondria ( $3 \sim 5 \mathrm{mg}$ protein) were suspended in $1.7 \mathrm{ml}$ of $0.25 \mathrm{M}$ mannitol, $70 \mathrm{~mm}$ sucrose, 1.0 mM phosphate, $5.0 \mathrm{mM}^{\mathrm{MgCl}}$ and $10 \mathrm{~mm}$ HEPESTEA, pH 7.4. With the exception of caprylate, all substrates were added at a final concentration of $10 \mathrm{~mm}$ with or without $1.0 \mathrm{mM}$ malate. Antibiotic W341C, ADP and FCCP concentrations were $1.0 \mu \mathrm{M}, 0.6 \mathrm{mM}$ and $1.0 \mu \mathrm{M}$, respectively. Parallel patterns were obtained using $1.0 \mu \mathrm{M}$ nigericin.

Table 3. Effect of W341C on mitochondrial adenine nucleotide translocase activity.

\begin{tabular}{lc}
\hline & $\begin{array}{c}\text { nmol ADP/ } \\
\text { mg protein }\end{array}$ \\
\hline Control & $0.651 \pm 0.133$ \\
Carboxyatractyloside $(50 \mu \mathrm{M})$ & 0 \\
W341C $(100 \mathrm{nM})$ & $0.727 \pm 0.129$ \\
Nigericin $(100 \mathrm{nM})$ & $0.561 \pm 0.043$ \\
\hline
\end{tabular}

In parallel experiments using $0.2 \mu \mathrm{Ci} / \mu \mathrm{mol}\left[{ }^{14} \mathrm{C}\right]-$ ADP or $12 \mu \mathrm{Ci} / \mu \mathrm{mol}\left[{ }^{14} \mathrm{C}\right]$ sucrose, mitochondria $\left(6.1 \mathrm{mg}\right.$ protein) were incubated at $0^{\circ} \mathrm{C}$ in $1.05 \mathrm{ml}$ of $0.25 \mathrm{M}$ mannitol, $70 \mathrm{~mm}$ sucrose, $1.0 \mathrm{~mm}$ EDTA, $0.6 \mathrm{~mm}$ ADP, $10 \mathrm{~mm}$ HEPES-TEA, $\mathrm{pH} 7.4$, and the agent indicated. After 30 seconds, carboxyatractyloside was added to a final concentration of $75 \mu \mathrm{M}$. The mitochondria were centrifuged for 1 minute at $8,000 \times g$ and the supernatant removed. The pellets were dissolved in $85 \%$ formic acid and the radioactivity counted in Aquasol in a liquid scintillation spectrophotometer. For tubes containing the same inhibitor, the $\left[{ }^{14} \mathrm{C}\right]$ sucrose $\mathrm{dpm}$ was used to correct for the $\left[{ }^{14} \mathrm{C}\right] \mathrm{ADP}$ dpm not within the matrix. Results are expressed as the mean $\pm S D$ of triplicates from one representative experiment.
Fig. 4. Effect of W341C on arsenate-uncoupled mitochondrial respiration.

Mitochondrial respiration was followed at $30^{\circ} \mathrm{C}$ in $2 \mathrm{ml}$ of $0.25 \mathrm{M}$ mannitol, $70 \mathrm{~mm}$ sucrose, $5.0 \mathrm{~mm}$ $\mathrm{MgCl}_{2}, 10.0 \mathrm{~mm}$ HEPES-TEA and $3.74 \mathrm{mg}$ mitochondrial protein. Arsenate and potassium values are from experiments run in parallel with respiration, but on a 2 -fold scale. Arsenate ( $2 \mathrm{~mm}$ final) and $\mathrm{W} 341 \mathrm{C}(1.0 \mu \mathrm{M}$ final) additions are indicated by arrows and occurred at 2.0 and 4.5 minutes, respectively. Reactions during the course of an experiment were terminated at discrete time points by centrifugation of aliquots removed from a common chamber. This procedure and the further processing of the mitochondrial pellet and supernatant fractions obtained upon centrifugation were performed as described in Materials and Methods. Symbols denote: Extramitochondrial potassium $(\triangle)$, intramitochondrial arsenate in the absence of W341C (O) and intramitochondrial arsenate in the presence of W341C (?). Similar results were obtained using $1.0 \mu \mathrm{M}$ nigericin.
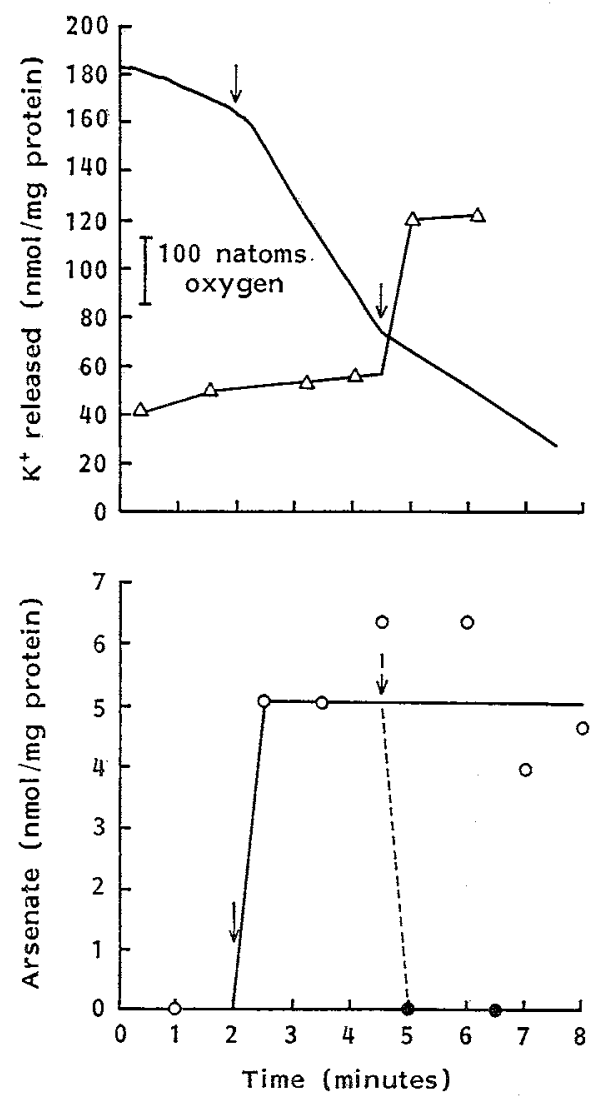
release was observed.

A comprehensive study of the effect of W341C on mitochondrial substrate oxidation revealed a pattern of inhibition similar to that previously reported for nigericin ${ }^{11,12)}$ (Table 2). Inhibition of state 3 respiration by W341C was observed with all substrates tested, while inhibition of FCCP-uncoupled respiration by $\mathrm{W} 341 \mathrm{C}$ was clearly substrate specific. In addition, only glutamate was able to reverse the ionophore-inhibited, FCCP-uncoupled oxidation of malate.

To investigate the inhibitory effect of monocarboxylic polyether antibiotics on state 3 respiration, a study was performed on the effect of W341C and nigericin on arsenate-uncoupled respiration. Arsenate enters the mitochondria by the phosphate carrier, ${ }^{13)}$ and like phosphate is a substrate for the mitochondrial ATP synthase. ${ }^{14)}$ The adenosine diphosphoarsenate formed hydrolyzes spontaneously to yield ADP and arsenate. ${ }^{14,15)}$ Thus, arsenate-uncoupled respiration is observed in the absence of added $\mathrm{ADP}^{16)}$ and should require only catalytic amounts of arsenate. The data presented in Fig. 4 demonstrate clearly the inhibitory effect of W341C on arsenate-uncoupled respiration. In addition, the respiratory inhibition caused by this antibiotic was concomitant with the release of both potassium and accumulated arsenate from the mitochondria (Fig. 4).

In a further study of state 3 inhibition by W341C and nigericin, the sensitivity of ADP uptake to inhibition by these agents was studied. As shown in Table 3, neither $\mathrm{W} 341 \mathrm{C}$ nor nigericin had a significant effect on adenine nucleotide translocase activity.

\section{Discussion}

Results from experiments on bulk phase ion transport and mitochondrial ion release indicate that antibiotic W341C is a monovalent cation ionophore with a preference for potassium transport. This finding is not unexpected, for structurally, antibiotic W341C is similar not only to the $\mathrm{K}^{+}$-ionophore nigericin, ${ }^{17)}$ but also to other $\mathrm{K}^{+}$-ionophores such as carriomycin, etheromycin and lonomycin $\mathrm{A} .{ }^{183}$ However, antibiotic W341C also bears close structural similarity to the $\mathrm{Mg}^{2+}$-selective ionophore 6016. ${ }^{19)}$ The presence of a C(2)-hydroxyl group in the otherwise monovalent ionophore-like structure of antibiotic- 6016 has been proposed as the major determinant of this ionophore's divalent cation complexing ability. ${ }^{20}$ ) Thus, the absence of a C(2)-hydroxyl moiety in antibiotic W341C probably explains this ionophore's inability to mediate $\mathrm{Mg}^{2+}$ and $\mathrm{Ca}^{2+}$ transport.

The inhibition of mitochondrial substrate oxidation by monocarboxylic polyether antibiotics is a well documented phenomenon. ${ }^{11,12}$ The inhibitory mechanism has been attributed to the ability of these antibiotics to mediate electroneutral alkali metal-proton exchange; ${ }^{21,22)}$ which in mitochondria results in perturbation of only the $\mathrm{pH}$ gradient component of the membrane potential. ${ }^{28)}$ Explanations for the inhibition of pyruvate, ${ }^{24)}$ malate, glutamate, and malate + pyruvate ${ }^{23)}$ oxidation, as well as the lack of inhibition of malate + glutamate, ${ }^{23)}$ succinate, ${ }^{233}$ glycerol-3-phosphate, ${ }^{252}$ and $\beta$-hydroxybutyrate ${ }^{26)}$ oxidation by these antibiotics all invoke either primarily or secondarily the impact of an altered mitochondrial $\mathrm{pH}$ gradient.

The inability of proline to reverse the ionophore-inhibited FCCP-uncoupled oxidation of malate implies that some aspect of proline metabolism prior to conversion to glutamate is inhibited. This inhibition could result from a perturbation of mitochondrial ion gradients by $\mathrm{W} 341 \mathrm{C}$ or nigericin, or by a route not related to the ionophoric activity of these compounds. However, in preliminary experiments (data not shown) no effect of nigericin was observed on the oxidation of proline by freezethawed mitochondria. Further work is needed to elucidate the mechanism by which W341C and nigericin inhibit proline metabolism.

Several factors may explain the inhibition of state 3 respiration by W341C and nigericin. LARDY et al. ${ }^{11)}$ and others ${ }^{23)}$ have proposed that the inhibition of $\beta$-hydroxybutyrate and succinate supported ATP synthesis by monovalent monocarboxylic polyether antibiotics is largely a result of the ability 
of these agents to prevent the accumulation of inorganic phosphate into mitochondria. In addition, PRESSMAN and LARDY established a potassium requirement for ATP synthesis, ${ }^{27)}$ but the $F_{1}-A T P a s e$ is not directly inhibited by nigericin. ${ }^{28)}$ Our data on the insensitivity of ADP uptake to W341C and nigericin effectively rule out the requirement of ADP supply as a site of ATP synthesis inhibition by these agents.

In an attempt to differentiate the requirement of a continuous inorganic phosphate supply for ATP synthesis from the potassium requirement, a study was performed on arsenate-uncoupled respiration. However, in arsenate-uncoupled mitochondria, the addition of either W341C or nigericin induced not only the loss of mitochondrial potassium, but also the loss of all measurable arsenate from the mitochondrial matrix space. Thus, while the inhibition of state 3 respiration by these antibiotics may be entirely due to the inhibition of phosphate accumulation, the role of potassium loss in this inhibition remains in question.

\section{Acknowledgment}

Supported by grants from the Mobil Foundation and NIH (AM. 10,334).

\section{References}

1) Wehrie, R. S.; C. Runsheng \& H. A. LARdy: The antibiotic W341C, its ion transport properties and inhibitory effects on mitochondrial substrate oxidation. Fed. Proc. 42:2829, 1983

2) SHU-XUN, L.; S. MEI-CHI \& W. JI-MIN: The fermentation, isolation and physico-chemical characterization of anticoccidal polyether W341C. In Fourth National Proceedings of the Symposium on Antibiotic Research. pp. 324 325, Shanghai Sciences and Technology Press, Shanghai, 1982

3) PeI-JUN, C.; W. JI-MIN \& C. JAU-ZUEN: Taxonomic study of Streptomyces W341 and antimicrobial action of its product W341C. In Fourth National Proceedings of the Symposium on Antibiotic Research. p. 324, Shanghai Sciences and Technology Press, Shanghai, 1982

4) Rungsheng, C. \& W. Ji-Min: Biochemical and biological characterization of ionophorous antibiotic W341C. Antibiotics (Chinese) 10: 342 347, 1985

5) Stru-Xun, L. \& S. MeI-CHI: Chemical structure of the new anticoccidal polyether W341C. In Fourth National Proceedings of the Symposium on Antibiotic Research. pp. 325 326, Shanghai Sciences and Technology Press, Shanghai, 1982

6) Pressman, B. C.: Properties of ionophores with broad range cation selectivity. Fed. Proc. 32: 1698 1703,1973

7) Johnson, D. \& H. A. LARdY: Isolation of liver or kidney mitochondria. In Methods in Enzymology. Vol. 10. Eds., R. EstabrooK \& M. Pullman, pp. 94 96, Academic Press, New York, 1967

8) LAYNE, E.: Spectrophotometric and turbidimetric methods for measuring proteins. In Methods in Enzymology. Vol. 3. Eds., S. Colowick \& N. KaplaN, pp. 447 454, Academic Press, New York, 1957

9) GUERIN, B.; M. GUERIN \& M. KLINGENBERG: Differential inhibition of phosphate effux and influx and a possible discrimination between an inner and outer location of the phosphate carrier in mitochondria. FEBS Lett. 10: 265 268, 1970

10) Lanzetta, P. A.; L. J. Alvarez, P. S. Reinach \& O. A. Candia: An improved assay for nanomole amounts of inorganic phosphate. Anal. Biochem. 100: 95 97, 1979

11) LARDY, H. A.; D. Johnson \& W. C. MCMurRaY: Antibiotics as tools for metabolic studies. I. A survey of toxic antibiotics in respiration, phosphorylative and glycolytic systems. Arch. Biochem. Biophys. 78: $587 \sim 597,1958$

12) CHA, C. M.: Inactivation of oxidation and phosphorylative systems in mitochondria by dianemycin and nigericin. Ph. D. Thesis, Univ. Wisconsin, 1962

13) Chappell, J. B.: Systems used for the transport of substrates into mitochondria. Br. Med. Bull. 24: $150 \sim 157,1968$

14) Gresser, M. J.: ADP-Arsenate. J. Biol. Chem. 256: 5981 5983, 1981

15) Lardy, H. A.; J. L. Connelly \& D. Johnson: Antibiotics as tools for metabolic studies. II. Inhibition of phosphoryl transfer in mitochondria by oligomycin and aurovertin. Biochemistry 3: 1961 1968, 1964

16) ERNSTER, L.; C.-P. LEE \& S. JANDA: The reaction sequence in oxidative phosphorylation. In Biochemistry of Mitochondria. Ed., E. C. SLATER et al., pp. 29 52, Academic Press, New York, 1967

17) Harned, R. L.; P. H. Hidy, C. J. CoRUm \& K. L. JoNEs: Nigericin, a new crystalline antibiotic from an 
unidentified streptomyces. Antibiot. Chemother. 1: 594 596, 1951

18) MItan, M. \& N. ÖTAKE: Studies on the ionophorous antibiotics. XV. The monovalent cation selective ionophorous activities of carriomycin, lonomycin and etheromycin. J. Antibiotics 31: 750 755, 1978

19) Mrtani, M. \& N. ŌTAKE: Ionophorous properties of antibiotic-6016, a novel magnesium selective ionophore. Agric. Biol. Chem. 43: 1543 1546, 1979

20) Ogita, T.; H. Nakayama, N. Ōtake, H. MiYamae, S. Sato \& Y. Saito: The crystal and molecular structure of the thallium salt of antibiotic-6016. Agric. Biol. Chem. 43:1537 1542, 1979

21) Pressman, B. C.; E. J. Harris, W. S. JAGGer \& J. H. Johnson: Antibiotic-mediated transport of alkali ions across lipid barriers. Proc. Natl. Acad. Sci. U.S.A. 58: 1949 1956, 1967

22) Lardy, H. A.; S. N. Graven \& S. Estrada-O: Specific induction and inhibition of cation and anion transport in mitochondria. Fed. Proc. 26: 1355 1360, 1967

23) Henderson, P. J.F.; J. D. McGivan \& J. B. Chappell: The action of certain antibiotics on mitochondrial, erythrocyte and artificial phospholipid membranes. The role of induced proton permeability. Biochem. J. 111 : $521 \sim 535,1969$

24) Williamson, J. R.: Role of anion transport in the regulation of metabolism. In Gluconeogenesis: Its Regulation in Mammalian Species. Eds., R. W. HaNson \& M. A. MemLMaN, pp. 165 220, John Wiley and Sons, New York, 1976

25) Klingenderg, M.: Localization of the glycerol-phosphate dehydrogenase in the outer phase of the mitochondrial inner membrane. Eur. J. Biochem. 13: 247 252, 1970

26) Ferguson, S. M. F.; S. Estrada-O \& H. A. LARdy: Potassium-specific uncoupling by nigericin. J. Biol. Chem. 246: 5645 5652, 1971

27) Pressman, B. C. \& H. A. Lardy: Further studies on the potassium requirements of mitochondria. Biochim. Biophys. Acta 18: 482 487, 1955

28) Estrada-O, S.; S. N. Graven \& H. A. Lardy: Potassium ion-dependent hydrolysis of adenosine triphosphate induced by nigericin in mitochondria. J. Biol. Chem. 242: 2925 2932, 1967 